\title{
Phonon excitations in the quasi-one-dimensional Haldane phase of $\mathrm{SrNi}_{2} \mathrm{~V}_{2} \mathrm{O}_{8}$
}

\author{
V. Kurnosov ${ }^{1}$, V. Gnezdilov ${ }^{1,2}$, P. Lemmens ${ }^{2}$, Yu. Pashkevich ${ }^{3}$, A.K. Bera ${ }^{4}$, \\ A.T.M.N. Islam ${ }^{4}$, and B. Lake ${ }^{4}$ \\ ${ }^{1}$ B. Verkin Institute for Low Temperature Physics and Engineering, NASU, Kharkov 61103, Ukraine \\ ${ }^{2}$ Institute for Condensed Matter Physics, TU Braunschweig, D-38106 Braunschweig, Germany \\ ${ }^{3}$ A.A. Galkin Donetsk Institute for Physics and Engineering, NASU, Kyiv 03680, Ukraine \\ ${ }^{4}$ Helmholtz-Zentrum Berlin, Hahn-Meitner-Platz 1, Berlin 14109, Germany \\ E-mail: kurnosov@ilt.kharkov.ua
}

Received April 4, 2017, published online October 25, 2017

\begin{abstract}
Vibrational Raman spectra of a single crystal of the coupled Haldane chain compound $\mathrm{SrNi}_{2} \mathrm{~V}_{2} \mathrm{O}_{8}$ with uniaxial anisotropy were investigated in the $10-1000 \mathrm{~cm}^{-1}$ frequency range at temperatures $7-300 \mathrm{~K}$. No structural phase transition was observed. The number of phonon lines observed in the experiment and their intensity were analyzed on the basis of the local symmetry considerations of different structural complexes. This approach was successful in explaining the discrepancy between the numbers of expected and experimentally observed phonon lines. Closeness of a real arrangement of some structural units to higher symmetry than the Wyckoff position results in strong interferential quenching of a number of Raman lines in the spectra.
\end{abstract}

PACS: 78.30.-j Infrared and Raman spectra;

63.20.-e Phonons in crystal lattices.

Keywords: Raman intensity, local symmetry consideration, interferential quenching.

\section{Introduction}

A remarkable feature of one-dimensional (1D) magnetic systems is the suppression of long-range magnetic order even at zero temperature by strong quantum spin fluctuations. Spin-1 Heisenberg antiferromagnetic (AFM) chains (Haldane chains) are of current interest due to their exotic magnetic properties [1,2]. The Haldane phase of integer spin chains has a unique many-body singlet ground state and gapped magnetic excitations $[3,4]$ in contrast to the gapless continuum of multispinon excitations of its half-integer counterpart, the spin-1/2 Heisenberg uniform AFM chain. The magnetic excitation spectra of an isolated Haldane chain were investigated in great detail using theoretical methods [5-7], as well as experimental techniques [8,9].

Raman scattering was proven to be a versatile tool to investigate compounds that represent low-dimensional quantum spin systems [10]. In addition to spin excitations, also excitations of the lattice system or even coupled modes can be investigated. This may help to better understand the effect of the lattice degrees of freedom on the spin system. Despite the great interest in Haldane chains materials, there are very few studies of these systems using Raman spectroscopy. To our present knowledge, detailed investigations only exist for the one $S=1$ spin chain compound $\mathrm{Y}_{2} \mathrm{BaNiO}_{5}$ [11].

In real systems the presence of interchain interactions and anisotropy leads to complex behaviors and a rich phase diagram as theoretically described in Ref. 12 . In this regard compounds $\mathrm{ANi}_{2} \mathrm{~V}_{2} \mathrm{O}_{8}$, where $\mathrm{A}=\mathrm{Sr}$ or $\mathrm{Pb}$, are of interest since they have substantial interchain interactions and single-ion uniaxial anisotropy [13,14]. According to earlier experiments on powder samples $[13,15]$ the ground state of $\mathrm{SrNi}_{2} \mathrm{~V}_{2} \mathrm{O}_{8}$ was suggested to be $3 \mathrm{D}$ ordered below $\sim 7 \mathrm{~K}$. Later, in numerous experiments [16-22] it has been convincingly shown that $\mathrm{SrNi}_{2} \mathrm{~V}_{2} \mathrm{O}_{8}$ has a nonmagnetic spinsinglet ground state.

Our present research activities aim to analyze phonon excitations of the quasi-1D Haldane compound $\mathrm{SrNi}_{2} \mathrm{~V}_{2} \mathrm{O}_{8}$ in detail. These are based on the study of the crystal symmetry and phonon excitations that in many cases help to uncover fundamental properties.

\section{Experimental}

Details of the $\mathrm{SrNi}_{2} \mathrm{~V}_{2} \mathrm{O}_{8}$ single crystals growth and characterization can be found in Refs. 19, 20. Our Raman scatter- 
ing experiments were performed in a quasi-backscattering geometry on $a b$ and $a c$ surfaces of $\mathrm{SrNi}_{2} \mathrm{~V}_{2} \mathrm{O}_{8}$ single crystals. A solid-state laser with the excitation wavelengths $\lambda=532.1 \mathrm{~nm}$ and a power level $P=5 \mathrm{~mW}$ was used for the spectra excitation. Spectra of the scattered radiation were collected by a Dilor-XY triple spectrometer and recorded by a liquid-nitrogen-cooled CCD detector (Horiba Jobin Yvon, Spectrum One CCD-3000V) with a spectral resolution of $<0.5 \mathrm{~cm}^{-1}$. In our experiments we used parallel $(a a, c c)$ and crossed $(a b, a c)$ light polarizations. Temperature dependences of the Raman spectra were measured in a variable temperature closed cycle cryostat (Oxford/Cryomech Optistat, RT-2.8 K).

\section{Phonon spectra}

\subsection{Group theoretical analysis and polarization rules}

The structure of $\mathrm{SrNi}_{2} \mathrm{~V}_{2} \mathrm{O}_{8}$ is described by the space group $I 4_{1} c d$ (\#110, $C_{4 v}^{12}$ ) with $Z=8$ formula units per unit cell $[20,23]$. According our knowledge, there is no evidence of structural instabilities for temperatures below room temperature. Due to the existence of the "rigid" ionic complexes $\left(\mathrm{VO}_{4}\right)^{3-}$ the vibrational modes may be expressed formally in terms of internal and external degrees of freedom of these extended complexes and with respect to degrees of freedom of the point ions $\mathrm{Sr}^{2+}$ and $\mathrm{Ni}^{2+}$ (see Table 1).

Table 1. Representation of $\mathrm{SrNi}_{2} \mathrm{~V}_{2} \mathrm{O}_{8}$ vibrational modes in terms of internal and external degrees of freedom of ionic complexes $\left(\mathrm{VO}_{4}\right)^{3-}$ and translations of point ions $\mathrm{Sr}^{2+}$ and $\mathrm{Ni}^{2+}$ using the space group $I 4_{1} c d$ and 8 formula units per unit cell

\begin{tabular}{|c|c|c|}
\hline \multicolumn{2}{|c|}{ Ions, modes } & Irreps \\
\hline \multirow{4}{*}{\multicolumn{2}{|c|}{$\begin{array}{c}\mathrm{Sr}^{2+} \text {, translational } \\
\mathrm{Ni}^{2+} \text {, translational } \\
\left(\mathrm{VO}_{4}\right)^{3-} \text {, translational } \\
\left(\mathrm{VO}_{4}\right)^{3-} \text {, rotational } \\
\end{array}$}} & $1 A_{1}+1 A_{2}+1 B_{1}+1 B_{2}+4 E$ \\
\hline & & $3 A_{1}+3 A_{2}+3 B_{1}+3 B_{2}+6 E$ \\
\hline & & $3 A_{1}+3 A_{2}+3 B_{1}+3 B_{2}+6 E$ \\
\hline & & $3 A_{1}+3 A_{2}+3 B_{1}+3 B_{2}+6 E$ \\
\hline \multicolumn{2}{|c|}{ Lattice optical } & $9 A_{1}+10 A_{2}+10 B_{1}+10 B_{2}+21 E$ \\
\hline \multicolumn{2}{|c|}{ Lattice acoustic } & $A_{1}+E$ \\
\hline \multirow{4}{*}{$\begin{array}{c}\left(\mathrm{VO}_{4}\right)^{3-}, \\
\text { internal }\end{array}$} & $v_{1}\left(A_{1}\right)$ & $1 A_{1}+1 A_{2}+1 B_{1}+1 B_{2}+2 E$ \\
\hline & $v_{2}(E)$ & $2 A_{1}+2 A_{2}+2 B_{1}+2 B_{2}+4 E$ \\
\hline & $v_{3}\left(F_{2}\right)$ & $3 A_{1}+3 A_{2}+3 B_{1}+3 B_{2}+6 E$ \\
\hline & $v_{4}\left(F_{2}\right)$ & $3 A_{1}+3 A_{2}+3 B_{1}+3 B_{2}+6 E$ \\
\hline \multicolumn{2}{|c|}{$\left(\mathrm{VO}_{4}\right)^{3-}$, internal (total) } & $9 A_{1}+9 A_{2}+9 B_{1}+9 B_{2}+18 E$ \\
\hline
\end{tabular}

There are 4 Raman-active irreps $A_{1}, B_{1}, B_{2}$, and $E$ whose polarizability tensors in coordinates corresponding to crystallographic directions of $4 \mathrm{~mm}\left(C_{4 v}\right)$ have the form

$$
\begin{array}{r}
A_{1}, z \rightarrow\left(\begin{array}{ccc}
a & & \\
& a & \\
& & b
\end{array}\right), B_{1} \rightarrow\left(\begin{array}{ll}
c & \\
& -c
\end{array}\right), B_{2} \rightarrow\left(d^{d}\right. \\
e, x \rightarrow\left(\begin{array}{l}
e \\
e
\end{array}\right), E, y \rightarrow\left(\begin{array}{c}
e \\
e
\end{array}\right)
\end{array}
$$

If orientations of $x$ and $y$ axes are not precisely determined, the above matrices, excluding fully symmetrical irrep $A_{1}$, may be generally rewritten as

$$
\begin{aligned}
& B_{1}(\theta) \rightarrow\left(\begin{array}{cc}
c \cos 2 \theta & c \sin 2 \theta \\
c \sin 2 \theta & -c \cos 2 \theta
\end{array}\right), \\
& B_{2}(\theta) \rightarrow\left(\begin{array}{cc}
-d \sin 2 \theta & d \cos 2 \theta \\
d \cos 2 \theta & d \sin 2 \theta
\end{array}\right) \\
& E, x(\theta) \rightarrow\left(\begin{array}{ll} 
& e \cos \theta \\
& e \sin \theta \\
e \cos \theta & e \sin \theta
\end{array}\right) \text {, } \\
& E, y(\theta) \rightarrow\left(\begin{array}{rrr} 
& -e \sin \theta \\
& e \cos \theta \\
-e \sin \theta & e \cos \theta &
\end{array}\right) \text {, }
\end{aligned}
$$

where $\theta$ is an angle between real crystallographic direction and arbitrary direction in the basal plane of the crystal. According to (2) the uncertainty in the orientation leads to a mixing of the polarization selection rules for Raman lines which belong to phonons with $B_{1}$ and $B_{2}$ symmetry. On the one hand, such mixing makes $B_{1}$ and $B_{2}$ modes unresolved, but, on the other hand, it helps to resolve $A_{1}$ and $B_{1}$ (and/or $B_{2}$ ) ones. Polarization $z z$ is "pure" for $A_{1}$ modes, all other are forbidden. It seems that this property is useful for the identification of $A_{1}$ type modes and their selection in other polarizations. But here we face some peculiarity: the polar character of the $A_{1}$ modes leads to a splitting of their longitudinal (LO) and transversal (TO) components.

In opaque crystals the observation of LO and TO components of polar phonons in Raman spectra depends on specifics of experimental back scattering geometry. This information is collected in Table 2.

Experimental Raman data were obtained using two sample orientations so that plane of incident light reflection contained $c_{4}$ axis of the crystal, or was perpendicular to it. Table 2 shows, for instance, that in $z z$ polarization only $A_{1}$ modes are present (and only TO components), while in $x^{\prime} x^{\prime}$ polarization, besides $B_{1}$ and $B_{2}$ phonons, $A_{1}$ modes are present (LO components). So, for $A_{1}$ modes possessing large LO-TO splitting $z z$ and $x^{\prime} x^{\prime}$ polarized spectra are different in the positions of the lines.

\subsection{Structure of the Raman spectrum and molecular vibrations of $\left(\mathrm{VO}_{4}\right)^{3-}$}

The free ion $\left(\mathrm{VO}_{4}\right)^{3-}$ is a tetrahedral complex and described within the $T_{d}$ point group. It possesses 9 internal degrees of freedom, which are grouped into 4 modes transformed as $A_{1}\left(v_{1}\right)+E\left(v_{2}\right)+2 F_{2}\left(v_{3}+v_{4}\right)$ irreps of $T_{d}$. Two of them belong to so-called symmetric $A_{1}\left(v_{1}\right)$ and asymmetric $F_{2}\left(v_{3}\right)$ stretchings of $\mathrm{V}-\mathrm{O}$ valence bonds. They have a priori the highest vibrational frequency. Two remaining 
Table 2. Raman selection rules for phonons of a different symmetry in the quasi-back scattering geometry utilized in the experiments. Symbols: $\mathbf{q}$ is direction of phonon wave-vector, $\mathbf{e}_{i}$ and $\mathbf{e}_{s}$ are polarizations of an incident and scattered light, respectively, $\alpha=\cos \theta, \beta=\sin \theta$. Intensities of a scattering are evaluated using the tensor components from (1)

\begin{tabular}{c|c|c|c|c|c|c}
\hline \hline $\mathbf{q}$ & $\mathbf{e}_{i}$ & $\mathbf{e}_{s}$ & $A_{1}$ & $E$ & $B_{1}$ & $B_{2}$ \\
\hline \hline$(0,0,1)$ & $(\alpha, \beta, 0)$ & $(\alpha, \beta, 0)$ & $a^{2}(\mathrm{LO})$ & & $\left(\alpha^{2}-\beta^{2}\right)^{2} c^{2}$ & $4(\alpha \beta)^{2} d^{2}$ \\
$(0,0,1)$ & $(\alpha, \beta, 0)$ & $(\beta,-\alpha, 0)$ & & $4(\alpha \beta)^{2} c^{2}$ & $\left(\alpha^{2}-\beta^{2}\right)^{2} d^{2}$ \\
$(\alpha, \beta, 0)$ & $(0,0,1)$ & $(0,0,1)$ & $b^{2}(\mathrm{TO})$ & & \\
$(\alpha, \beta, 0)$ & $(\beta,-\alpha, 0)$ & $(0,0,1)$ & & $e^{2}(\mathrm{TO})$ & & \\
\hline \hline
\end{tabular}

vibrational modes of the $\left(\mathrm{VO}_{4}\right)$ tetrahedron are the symmetric $E\left(v_{2}\right)$ and asymmetric $F_{2}\left(v_{4}\right)$ bendings of the valence angles. Their frequencies are very close in the free complex and are more than twice less than the frequency of stretching mode $v_{1}$. In crystals the characteristic frequency ranges of these modes are: $A_{1}\left(v_{1}\right)-800-900, E\left(v_{2}\right)-300-450$, $F_{2}\left(v_{3}\right)-700-900, F_{2}\left(v_{4}\right)-300-450 \mathrm{~cm}^{-1}$ [24-30]. In aqueous solutions of $\left(\mathrm{VO}_{4}\right)^{3-}$ containing salts the frequencies are: $A_{1}\left(v_{1}\right)-826 \pm 1, F_{2}\left(v_{3}\right)-(804 \pm 4) \mathrm{cm}^{-1}, E\left(v_{2}\right)$ and $F_{2}\left(v_{4}\right)-(336 \pm 2) \mathrm{cm}^{-1}$ [31].

The $\mathrm{O}^{2-}$ ion has a large enough ionic radius of $\sim 1.38 \AA$ [32], so oxides structures often may be described like a lattice of these close packed ions, voids between which are occupied by "small” metallic ions. $\mathrm{SrNi}_{2} \mathrm{~V}_{2} \mathrm{O}_{8}$ has quite such structure, where the octahedral surrounding of every $\mathrm{Ni}^{2+}$ ion consists of the same $\mathrm{O}^{2-}$ ions that belong to six different $\left(\mathrm{VO}_{4}\right)^{3-}$ complexes. Octahedral oxygen complexes of transitional metals are very popular units representing metal-oxide crystal structures too. The internal vibrations of $\left(\mathrm{NiO}_{6}\right)$ octahedrons reach frequencies of about $550 \mathrm{~cm}^{-1}$ [33-35], so the individuality of all internal modes of $\left(\mathrm{VO}_{4}\right)^{3-}$ tetrahedra becomes doubtful. Looking on the Raman spectra of $\mathrm{SrNi}_{2} \mathrm{~V}_{2} \mathrm{O}_{8}$ one can divide them into two ranges: $50-500$ and $700-950 \mathrm{~cm}^{-1}$ (Fig. 1). It is clear that the high-frequency region is occupied by vibrations that originate from $A_{1}\left(v_{1}\right)$ and $F_{2}\left(v_{3}\right)$ internal modes of $8\left(\mathrm{VO}_{4}\right)^{3-}$ tetrahedra contained in the primitive cell. Only these modes keep their individuality and are well isolated from the low-frequency "lattice" vibration region. In contrast the bending modes of $\left(\mathrm{VO}_{4}\right)^{3-}$ tetrahedra merge with other modes of the crystal, producing the so-called "lattice" region. According to the Table 1, high-frequency region of the $\mathrm{SrNi}_{2} \mathrm{~V}_{2} \mathrm{O}_{8}$ Raman spectrum must contain lines corresponding to $4 A_{1}+4 B_{1}+4 B_{2}+8 E$ irreps ( $4 A_{2}$ irreps are silent). The lattice range corresponds to $14 A_{1}+15 B_{1}+15 B_{2}+31 E$ Raman-active vibrational modes, accordingly.

Let us consider the spectral features of the highfrequency region: Spectra in all experimental polarizations at low temperature are presented in Fig. 2. The decomposition into the series of Lorentz-shape-like spectral lines is presented ibid. At low temperatures the most intensive $A_{1}$ lines show an appreciable asymmetry on the highfrequency slope. The high-temperature spectra don't reveal such features due to a temperature induced broadening of the lines. The nature of such asymmetry is not in the center of the present paper. As a suitable hypothesis we propose, that it may be a result of the combination of intensive optical modes with acoustical modes. Asymmetry in this case reflects a difference between the probabilities to excite and annihilate acoustic phonons, which is larger at low temperatures. High temperature equalizes the probabilities and makes the wings of the line symmetrical.
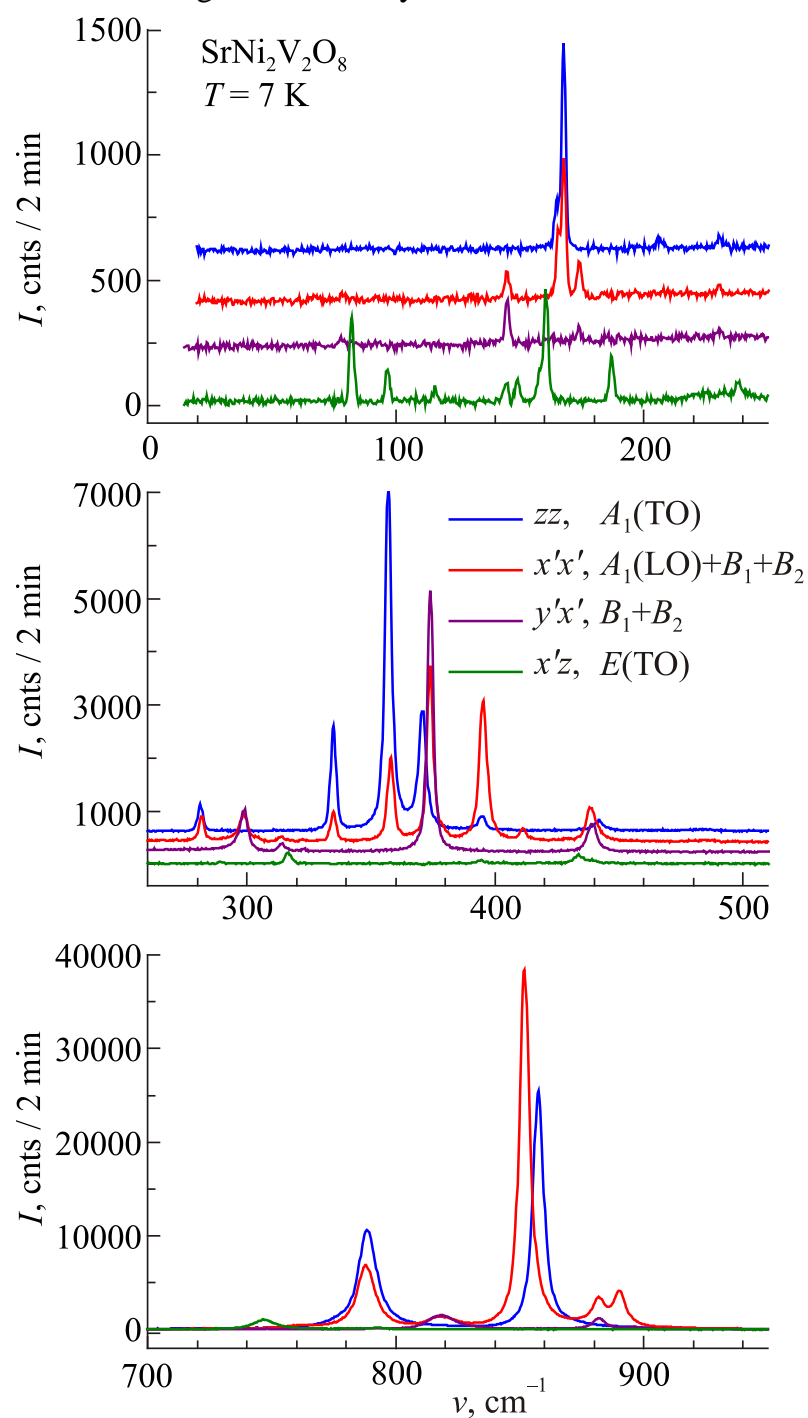

Fig. 1. (Color online) Raman spectra of $\mathrm{SrNi}_{2} \mathrm{~V}_{2} \mathrm{O}_{8}$ single crystal at $T=7 \mathrm{~K}$ in four polarizations. Irreps of Raman-active phonon excitations for every polarization are indicated. 

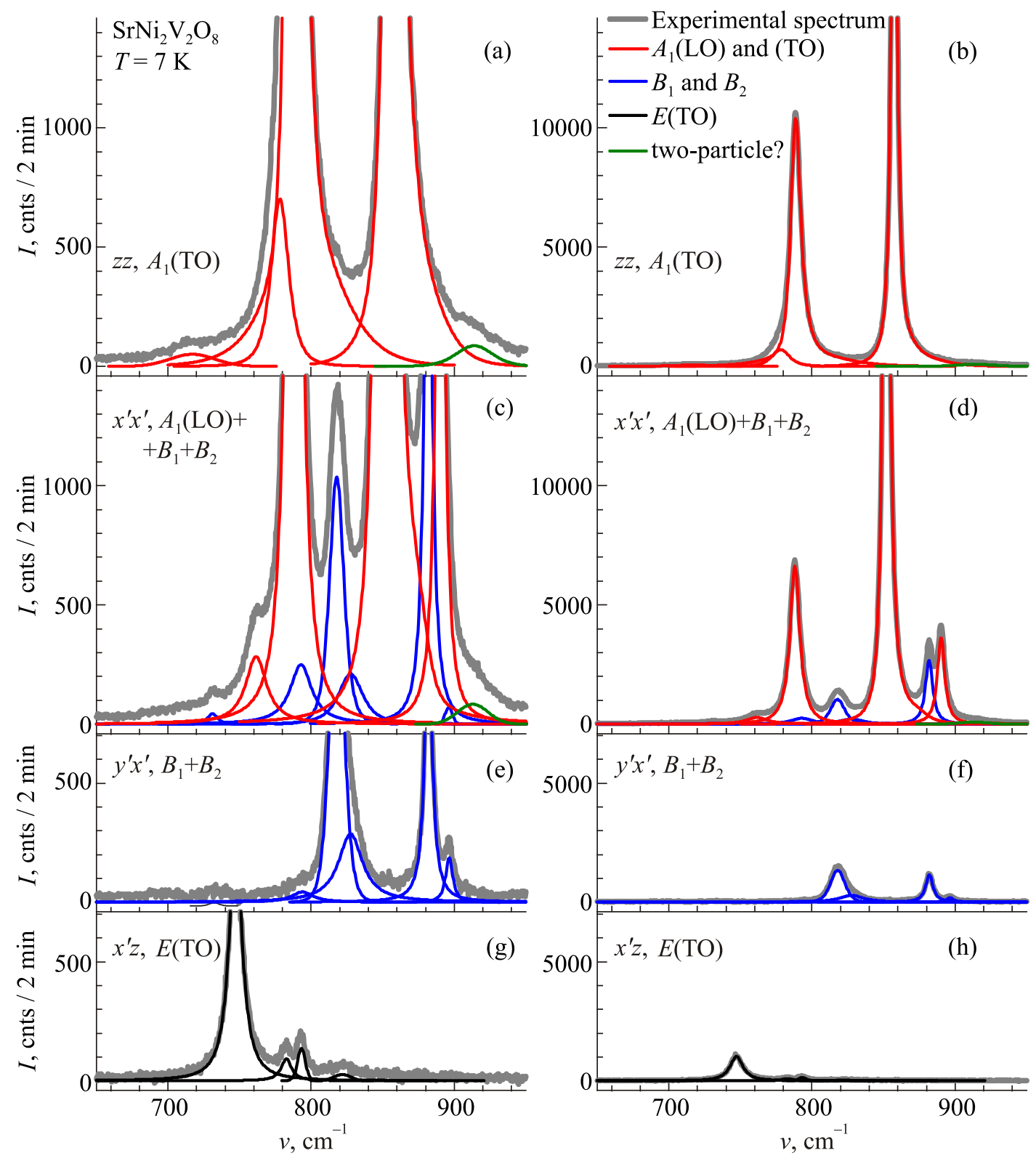

Fig. 2. (Color online) High-frequency Raman spectra of $\mathrm{SrNi}_{2} \mathrm{~V}_{2} \mathrm{O}_{8}$ in the range of $v_{1}\left(A_{1}\right)$ and $v_{3}\left(F_{2}\right)$ vibrational modes of $\left(\mathrm{VO}_{4}\right)^{3-}$ complex. Polarization and corresponding symmetry of the modes are marked. Intensity scales on left and right panels differ in 10 times.

All identified lines are collected in Table 3. The spectrum in $\mathrm{zz}$ polarization with $A_{1}(\mathrm{TO})$ consists of 5 lines, four of which are the Davydov components of $v_{1}\left(A_{1}\right)$ and $v_{3}\left(F_{2}\right)$ stretching modes of the $\left(\mathrm{VO}_{4}\right)^{3-}$ tetrahedron (Fig. 2(a), (b)). Note, that triple degeneracy of $v_{3}\left(F_{2}\right)$ mode is lifted due to crystal field effect. The most intense line at $857.5 \mathrm{~cm}^{-1}$ must be attributed to the $v_{1}$ mode according to frequency position. So, the highest-frequency line at $914 \mathrm{~cm}^{-1}$ most likely refers to some kind of two-particle excitation. The identical line is situated in $x^{\prime} x^{\prime}$ spectrum and has zero LO-TO splitting (Fig. 2(c), (d)). This spectrum contains a set of lines belonging to $A_{1}(\mathrm{LO}), B_{1}$, and $B_{2}$ modes. $B$ modes may be identified by means of a $y^{\prime} x^{\prime}$ polarized spectrum (Fig. 2(e), (f)). Remaining spectrum of $x^{\prime} z$ polarization contains lines related to $E(\mathrm{TO})$ modes.
The most intense line $\left(v_{1}\right)$ is observed in diagonal polarizations $z z$ and $x^{\prime} x^{\prime}$. It demonstrates a so-called "inverted” LO-TO splitting (see Table 3). Originally, in the frame of $T_{d}$ symmetry of a free $\left(\mathrm{VO}_{4}\right)^{3-}$ complex, this mode is nonpolar. It is in agreement with a small observed LO-TO splitting. Inversion is a result of a fall of its energy into a wide, forbidden gap of some strong dipolar mode. The same "inversion", but with smaller splitting is inherent in the other intensive line $\left(v_{3}\right)$ at $788 \mathrm{~cm}^{-1}$. Most probably the dipole mode with great oscillator strength originates from a polar $v_{3}$ mode of a $\left(\mathrm{VO}_{4}\right)_{-1}^{3-}$ tetrahedron and possesses 778.2 (TO)-890.2 (LO) $\mathrm{cm}^{-1}$ splitting that covers the greater part of discussed spectral range. As was mentioned above, every internal degree of freedom, taking into account its degeneracy, produces 4 different one-dimensional irreps 
Table 3. Frequencies, integral intensities and assignment of Raman lines observed in the spectral range of $v_{1}\left(A_{1}\right)$ and $v_{3}\left(F_{2}\right)$ vibrational modes of $\left(\mathrm{VO}_{4}\right)^{3-}$ complex in $\mathrm{SrNi}_{2} \mathrm{~V}_{2} \mathrm{O}_{8}$

\begin{tabular}{|c|c|c|c|c|}
\hline Frequency, $\mathrm{cm}^{-1}$ & $\begin{array}{l}\text { Integral intensity, } \\
\text { arb. units }\end{array}$ & Irreps & Polarization & Mode \\
\hline 717.0 & 1991 & $A_{1}(\mathrm{TO})$ & $z Z$ & \multirow{5}{*}{$v_{3}$} \\
\hline 761.6 & 7832 & $A_{1}(\mathrm{LO})$ & $x^{\prime} x^{\prime}$ & \\
\hline \multirow{2}{*}{731.5} & 451 & $B_{1}+B_{2}$ & $x^{\prime} x^{\prime}$ & \\
\hline & 201 & $B_{1}+B_{2}$ & $y^{\prime} x^{\prime}$ & \\
\hline 747.2 & 16606 & $E(\mathrm{TO})$ & $x^{\prime} z$ & \\
\hline 778.2 & 12734 & $A_{1}(\mathrm{TO})$ & $\mathrm{zz}$ & \\
\hline 890.2 & 33888 & $A_{1}(\mathrm{LO})$ & $x^{\prime} x^{\prime}$ & \\
\hline 782.7 & 1271 & $E(\mathrm{TO})$ & $x^{\prime} z$ & \\
\hline 793.2 & 958 & $E(\mathrm{TO})$ & $x^{\prime} z$ & $v_{3}$ \\
\hline \multirow{2}{*}{793.4} & 7188 & $B_{1}+B_{2}$ & $x^{\prime} x^{\prime}$ & \\
\hline & 1236 & $B_{1}+B_{2}$ & $y^{\prime} x^{\prime}$ & \\
\hline 788.0 & 80697 & $A_{1}(\mathrm{LO})$ & $x^{\prime} x^{\prime}$ & \multirow{7}{*}{$v_{3}$} \\
\hline 788.4 & 138057 & $A_{1}(\mathrm{TO})$ & $z Z$ & \\
\hline 8179 & 14509 & $B_{1}+B_{2}$ & $x^{\prime} x^{\prime}$ & \\
\hline $81 / .9$ & 17922 & $B_{1}+B_{2}$ & $y^{\prime} x^{\prime}$ & \\
\hline 821.7 & 387 & $E(\mathrm{TO})$ & $x^{\prime} z$ & \\
\hline \multirow{2}{*}{827.5} & 5594 & $B_{1}+B_{2}$ & $x^{\prime} x^{\prime}$ & \\
\hline & 9116 & $B_{1}+B_{2}$ & $y^{\prime} x^{\prime}$ & \\
\hline 851.9 & 282998 & $A_{1}(\mathrm{LO})$ & $x^{\prime} x^{\prime}$ & \multirow{6}{*}{$v_{1}$} \\
\hline 857.5 & 196273 & $A_{1}(\mathrm{TO})$ & $z z$ & \\
\hline 8819 & 24959 & $B_{1}+B_{2}$ & $x^{\prime} x^{\prime}$ & \\
\hline & 11177 & $B_{1}+B_{2}$ & $y^{\prime} x^{\prime}$ & \\
\hline \multirow{2}{*}{896.3} & 445 & $B_{1}+B_{2}$ & $x^{\prime} x^{\prime}$ & \\
\hline & 1585 & $B_{1}+B_{2}$ & $y^{\prime} x^{\prime}$ & \\
\hline 914.0 & 2902 & $A_{1}(\mathrm{TO})$ & $z z$ & \multirow{2}{*}{ two-particle } \\
\hline 912.9 & 2241 & $A_{1}(\mathrm{LO})$ & $x^{\prime} x^{\prime}$ & \\
\hline
\end{tabular}

$\left(A_{1}, A_{2}, B_{1}, B_{2}\right)$ and 2 two-dimensional ones $(E)$ in the crystal. So, there are 4 groups of lines, each belongs to one degree of freedom, and must be presented by $A_{1}+B_{1}+B_{2}+2 E$ symmetry. Figure 2 and Table 3 reveal detection of all $4 A_{1}$ lines, $6 B_{1}+B_{2}$ lines versus 8 allowed, and $4 E$ lines versus 8 allowed.

\subsection{Low-frequency Raman spectra of $\mathrm{SrNi}_{2} \mathrm{~V}_{2} \mathrm{O}_{8}$}

The low-frequency "lattice” range of Raman spectra does not show noticeable anomalies in their temperature dependence. Spectra in four polarizations belonging to phonons of different symmetry are presented in Fig. 3. The temperature dependences of the frequencies of all detected lines are shown in Fig. 4. A list of all detected phonon lines at lowest temperature with the indication of their symmetry and related polarization rules are given in Table 4 . The total number of observed and detected low-temperature lines is essentially smaller than the group theory predicts. Only 11 from $14 A_{1}, 15$ from $30\left(B_{1}+B_{2}\right)$, and 16 from $31 E$ of allowed phononic lines are detected. The cause of such a deficit is probably the big number of formula units in the crystal unit cell that leads to small Raman intensities of some modes due to practically full interferential quenching. These aspects are considered in the following section.

\section{Symmetry aspects of the phonon excitations intensity in the Raman spectrum of $\mathrm{SrNi}_{2} \mathrm{~V}_{2} \mathrm{O}_{8}$}

Intensity of the Raman scattering originates from a polarizability induced by an appropriate mode in the crystal. Group theoretical analysis gives the total number of the modes based on the symmetry positions of the ions in the structure. Symmetry of some complexes (dimensions, angles, etc.) of a crystal structure sometimes appears higher than their Wyckoff positions. It may lead to the extremely weak intensity of some Raman signals. The following sections cover these issues. 

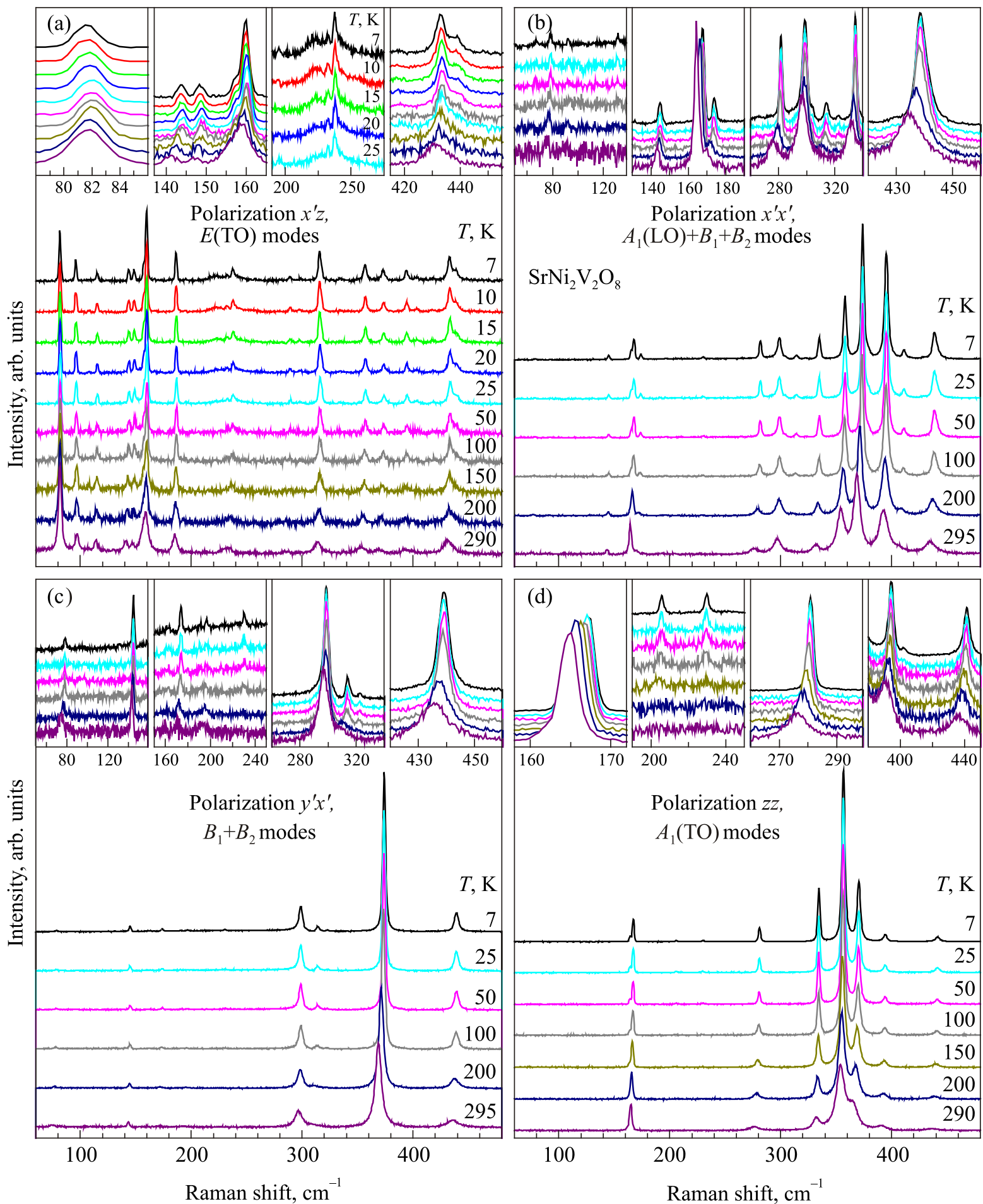

Fig. 3. (Color online) Temperature dependences of low-frequency range of $\mathrm{SrNi}_{2} \mathrm{~V}_{2} \mathrm{O}_{8}$ Raman spectrum in: (a) $x^{\prime} z$ polarization corresponding to $E(\mathrm{TO})$ modes, (b) $x^{\prime} x^{\prime}$ polarization corresponding to $A_{1}(\mathrm{LO})+B_{1}+B_{2}$ modes, (c) $y^{\prime} x^{\prime}$ polarization corresponding to $B_{1}+B_{2}$ modes, and (d) zz polarization corresponding to $A_{1}(\mathrm{TO})$ modes. Insets show some spectral peculiarities in detail.

\section{1. $\left(\mathrm{VO}_{4}\right)^{3-}$ modes}

In the structure of $\mathrm{SrNi}_{2} \mathrm{~V}_{2} \mathrm{O}_{8}$ the $\left(\mathrm{VO}_{4}\right)^{3-}$ complexes occupy general positions, but their local second order axes in the $T_{d}$ symmetry notation are oriented close to the principal axes of the crystal. More precisely, there are two groups of the tetrahedra whose second order axes are prac- tically parallel to $x$ and $y$ crystallographic directions. Furthermore, the tetrahedra are slightly rotated around these preferred axes as can be seen in Fig. 5. We also consider some uniaxial distortion of the tetrahedra along those preferred axes in order to include to their polarizability some small parameters reflected the low symmetry of the position. 


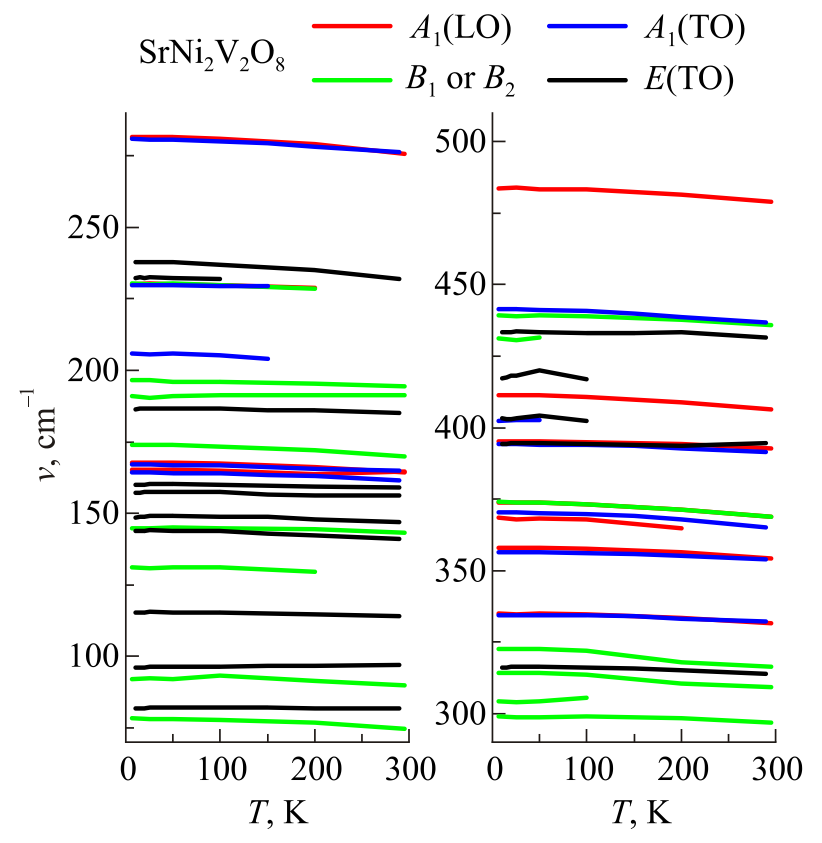

Fig. 4. (Color online) Temperature dependences of frequencies of all detected Raman-active phonons in the low-frequency range in $\mathrm{SrNi}_{2} \mathrm{~V}_{2} \mathrm{O}_{8}$.

Using such approach, the polarizabilities of an individual $\mathrm{VO}_{4}$ tetrahedron related with its own modes in the crystal are expressed in the following manner:

$\alpha_{\mathrm{mol}}^{A_{1}}\left(A_{1}\right)=\left(\begin{array}{ccc}a+\Delta_{a} & & \\ & a & \\ & & a\end{array}\right), \quad \alpha_{\mathrm{mol}}^{A_{1}}(E(1))=\left(\begin{array}{lll}-2 b & \\ & b & \\ & & b\end{array}\right)$,

$\alpha_{\mathrm{mol}}^{B_{1}}(E(2))=\left(\begin{array}{ccc}0 & & \\ \sqrt{3} b & \delta_{b} \\ \delta_{b} & -\sqrt{3} b\end{array}\right), \alpha_{\mathrm{mol}}^{B_{2}(x)}\left(F_{2}(x)\right)=\left(\begin{array}{cc}\delta_{c} & c \\ c & -\delta_{c}\end{array}\right)$,

$\alpha_{\mathrm{mol}}^{E(y)}\left(F_{2}(y)\right)=\left(\begin{array}{c}\delta_{d} d \\ \delta_{d} \\ d\end{array}\right), \alpha_{\mathrm{mol}}^{E(z)}\left(F_{2}(z)\right)=\left(\begin{array}{r}d-\delta_{d} \\ d^{d} \\ -\delta_{d}\end{array}\right)$,

where term $\Delta_{a}$ and difference between $c$ and $d$ are due to an uniaxial distortion of the tetrahedron along $x$ axis; terms $\delta_{b}, \delta_{c}$, and $\delta_{d}$ are due to slight rotation around $x$ axis. Using these assumptions and symmetry coordinates of
Table 4. Frequencies of observed Raman lines $\left(\right.$ in $\mathrm{cm}^{-1}$ ) of the low-frequency spectral range in $\mathrm{SrNi}_{2} \mathrm{~V}_{2} \mathrm{O}_{8}$ at a temperature $7 \mathrm{~K}$. A symmetry assignment is based on the polarization selection rules

\begin{tabular}{|c|c|c|c|}
\hline$A_{1}(\mathrm{TO})$ & $A_{1}(\mathrm{LO})$ & $B_{1}+B_{2}$ & $E(\mathrm{TO})$ \\
\hline \multirow[t]{8}{*}{$\mathrm{zz}$} & $x^{\prime} x^{\prime}$ & $x^{\prime} x^{\prime}, y^{\prime} x^{\prime}$ & $x^{\prime} z$ \\
\hline & & 78.5 & 81.7 \\
\hline & & 92.0 & 96.1 \\
\hline & & 131.2 & 115.2 \\
\hline & & 144.9 & 143.8 \\
\hline & & & 148.6 \\
\hline & & & 157.1 \\
\hline & & & 159.9 \\
\hline 164.3 & 165.3 & & \\
\hline \multirow[t]{3}{*}{167.2} & 167.8 & & \\
\hline & & 173.9 & \\
\hline & & 190.9 & 186.5 \\
\hline 205.8 & & 196.7 & \\
\hline \multirow[t]{3}{*}{229.9} & 230.2 & 230.4 & \\
\hline & & & 232.3 \\
\hline & & & 238.0 \\
\hline \multirow[t]{4}{*}{280.9} & 281.8 & & 289.2 \\
\hline & & 298.9 & \\
\hline & & 304.2 & \\
\hline & & 314.3 & 316.1 \\
\hline 334.5 & 334.9 & 322.7 & \\
\hline 356.6 & 358.0 & & \\
\hline 370.5 & 368.4 & 374.0 & \\
\hline 394.3 & 395.3 & & 394.3 \\
\hline \multirow[t]{3}{*}{402.3} & 411.3 & & 403.3 \\
\hline & & & 417.2 \\
\hline & & 431.1 & 433.2 \\
\hline 441.5 & 483.7 & 439.2 & \\
\hline
\end{tabular}

nondegenerated modes of the crystal, the polarizabilities arising from $A_{1}, E$, and $F_{2}$ modes of the tetrahedra can be expressed as follows:

$$
\begin{aligned}
& \alpha_{\text {cryst }}^{A_{1}}\left(A_{1}\right)=\sqrt{2}\left(\begin{array}{lll}
2 a+\Delta_{a} & \\
& 2 a+\Delta_{a} & \\
& & 2 a
\end{array}\right), \\
& \alpha_{\text {cryst }}^{A_{1}}(E(1))=\sqrt{2}\left(\begin{array}{lll}
-b & \\
& -b & \\
& & 2 b
\end{array}\right),
\end{aligned}
$$$$
\begin{aligned}
& \alpha_{\text {cryst }}^{B_{1}}\left(A_{1}\right)=\sqrt{2}\left(\begin{array}{ccc}
\Delta_{a} & & \\
& -\Delta_{a} & \\
& & 0
\end{array}\right), \\
& \alpha_{\text {cryst }}^{B_{1}}(E(1))=3 \sqrt{2}\left(\begin{array}{lll}
-b & & \\
& b & \\
& & 0
\end{array}\right),
\end{aligned}
$$ 


$$
\begin{aligned}
& \alpha_{\text {cryst }}^{A_{1}}(E(2))=\sqrt{6}\left(\begin{array}{lll}
b & & \\
& b & \\
& & -2 b
\end{array}\right) \text {, } \\
& \alpha_{\text {cryst }}^{B_{1}}(E(2))=\sqrt{6}\left(\begin{array}{lll}
b & & \\
& -b & \\
& & 0
\end{array}\right) \text {, } \\
& \alpha_{\text {cryst }}^{A_{1}}\left(F_{2}(x)\right)=\sqrt{2}\left(\begin{array}{llll}
\delta_{c} & & \\
& \delta_{c} & \\
& & -2 \delta_{c}
\end{array}\right) \text {, } \\
& \alpha_{\text {cryst }}^{B_{1}}\left(F_{2}(x)\right)=\sqrt{2}\left(\begin{array}{lll}
-\delta_{c} & & \\
& \delta_{c} & \\
& & 0
\end{array}\right) \text {, } \\
& \alpha_{\text {cryst }}^{B_{2}}\left(F_{2}(y)\right)=2 \sqrt{2}\left(\begin{array}{cc} 
& \delta_{d} \\
\delta_{d} &
\end{array}\right) \text {, } \\
& \alpha_{\text {cryst }}^{B_{2}}\left(F_{2}(z)\right)=2 \sqrt{2}\left(d^{d}\right) \text {. }
\end{aligned}
$$

Here the upper indices mark irreps of the crystal modes while symbols in parentheses belong to the internal modes of tetrahedron.

Twice-degenerated modes of the crystal have $x z$ and $y z$ components of polarizability tensor only. That is why they can only originate from those modes of an individual molecule, whose polarizabilities contain such components. It follows from (3) that appreciable Raman intensity of twice degenerated modes in the crystal arises from the modes related to $E(2), F_{2}(x), F_{2}(y)$, and $F_{2}(z)$ molecular vibrations only. Theoretically these 4 degrees of freedom generate 8 crystal $E$ modes, but only half of them possess nonzero Raman polarizability. In order to understand why this happens we can consider the example of $F_{2}(x)$ and $F_{2}(y)$ molecular degrees of freedom. These modes are dipolar that makes convenient their vector representation, as shown in Fig. 5. The $x z$ components of crystal polarizability for modes shown in Fig. 5 are expressed as follows according to (4):

$$
\begin{aligned}
& \alpha_{\text {cryst }}^{x z}\left(S_{1}\right)=\frac{1}{2 \sqrt{2}}\left(\alpha_{1}^{y z}+\alpha_{4}^{y z}+\alpha_{6}^{y z}+\alpha_{7}^{y z}+\alpha_{2}^{x z}+\alpha_{3}^{x z}+\alpha_{5}^{x z}+\alpha_{8}^{x z}\right)=\sqrt{2}(c+d), \\
& \alpha_{\text {cryst }}^{x z}\left(S_{2}\right)=\frac{1}{2 \sqrt{2}}\left(\alpha_{1}^{y z}+\alpha_{4}^{y z}+\alpha_{6}^{y z}+\alpha_{7}^{y z}-\alpha_{2}^{x z}-\alpha_{3}^{x z}-\alpha_{5}^{x z}-\alpha_{8}^{x z}\right)=\sqrt{2}(c-d) \text {, } \\
& \alpha_{\text {cryst }}^{x z}\left(S_{3}\right)=\frac{1}{2 \sqrt{2}}\left(\alpha_{1}^{y z}-\alpha_{4}^{y z}-\alpha_{6}^{y z}+\alpha_{7}^{y z}+\alpha_{2}^{x z}-\alpha_{3}^{x z}-\alpha_{5}^{x z}+\alpha_{8}^{x z}\right)=0, \\
& \alpha_{\text {cryst }}^{x z}\left(S_{4}\right)=\frac{1}{2 \sqrt{2}}\left(\alpha_{1}^{y z}-\alpha_{4}^{y z}-\alpha_{6}^{y z}+\alpha_{7}^{y z}-\alpha_{2}^{x z}+\alpha_{3}^{x z}+\alpha_{5}^{x z}-\alpha_{8}^{x z}\right)=0 .
\end{aligned}
$$
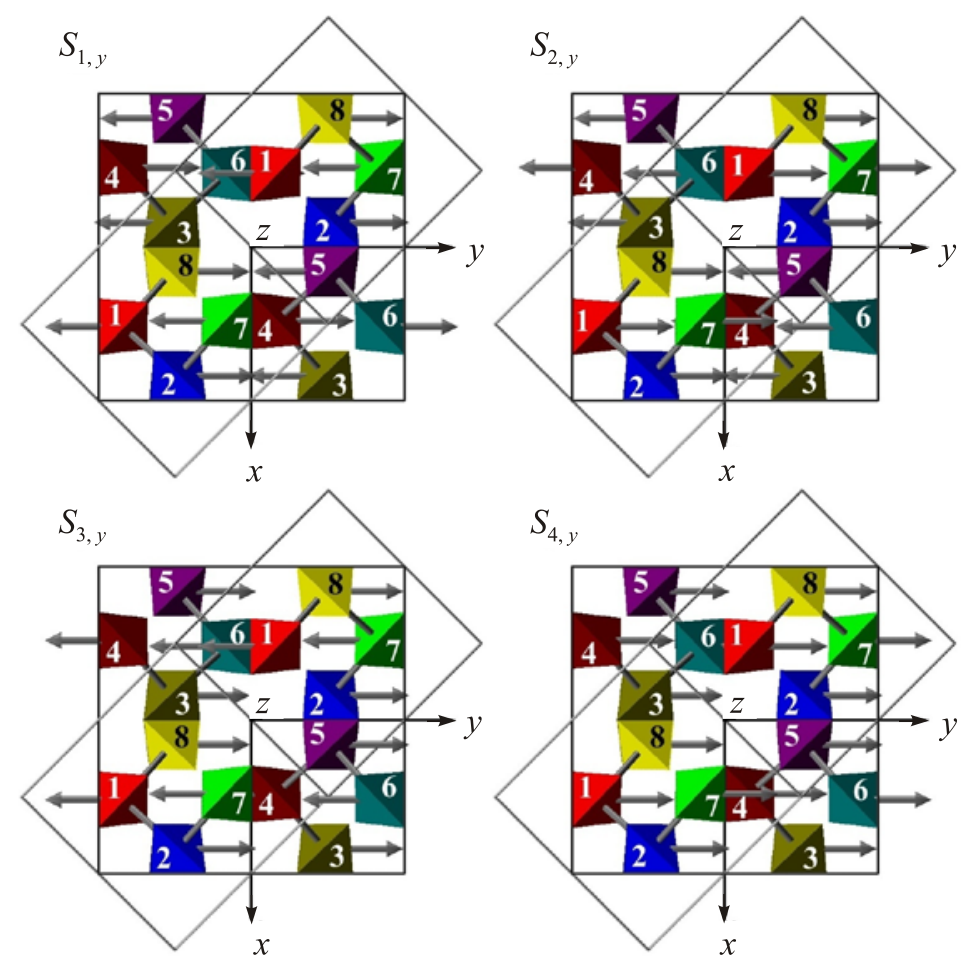

Fig. 5. (Color online) Patterns of $y$ components of twice-degenerated modes in $\mathrm{SrNi}_{2} \mathrm{~V}_{2} \mathrm{O}_{8}$ originating from $F_{2}$ vibrational modes of tetrahedral $\left(\mathrm{VO}_{4}\right)^{3-}$ complexes. Numeration of all tetrahedra belonging to primitive cell is used in expressions (5). 
Modes $S_{3}$ and $S_{4}$ are silent due to orientation of one of the second order axes of the tetrahedra along crystallographic direction in the crystal basal plane. Mode $S_{2}$ is also weak because $c \approx d$. All nonzero polarizability tensors of the crystal for twice-degenerated modes are presented below:

$$
\begin{aligned}
& \alpha_{\text {cryst }}^{E(x)}(E(2))=2\left(\begin{array}{r}
\delta_{b} \\
\delta_{b}
\end{array}\right), \\
& \alpha_{\text {cryst }}^{E(x)}\left(F_{2}(z)\right)=2\left(\begin{array}{ll}
-\delta_{d} \\
-\delta_{d}
\end{array}\right), \\
& \alpha_{\text {cryst }}^{E(x)}\left(F_{2}(x, y)\right)=\sqrt{2}\left(\begin{array}{ll}
d+c & d+c \\
d+c &
\end{array}\right), \\
& \alpha_{\text {cryst }}^{E(x)}\left(F_{2}(x, y)\right)=\sqrt{2}\left(\begin{array}{ll}
d-c & d-c \\
d &
\end{array}\right),
\end{aligned}
$$

Results of the analysis are collected in Table 5.

With regard to $A_{1}\left(v_{1}\right)$ and $F_{2}\left(v_{3}\right)$ modes of highfrequency region, only one $A_{1}\left(v_{1}\right)$, one $B_{2}\left(v_{3}\right)$, and one $E\left(v_{3}\right)$ modes are expected to be intense if we assume that their polarizability is originated purely from the molecular polarizability. Taking into account small deviations from ideal form and orientation of the tetrahedra the weak

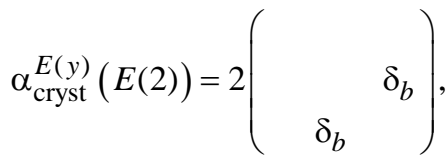

$$
\begin{aligned}
& \alpha_{\text {cryst }}^{E(y)}\left(F_{2}(z)\right)=2\left(\begin{array}{ll} 
& -\delta_{d} \\
& -\delta_{d}
\end{array}\right), \\
& \alpha_{\text {cryst }}^{E(y)}\left(F_{2}(x, y)\right)=\sqrt{2}\left(\begin{array}{ll} 
& d+c \\
d+c &
\end{array}\right), \\
& \alpha_{\text {cryst }}^{E(y)}\left(F_{2}(x, y)\right)=\sqrt{2}\left(\begin{array}{ll} 
& d-c \\
d-c &
\end{array}\right) .
\end{aligned}
$$

$A_{1}\left(v_{3}\right), B_{1}\left(v_{1}\right), B_{1}\left(v_{3}\right), B_{2}\left(v_{3}\right)$, and $2 E\left(v_{3}\right)$ can be added. A comparison with experiment shows partial match. So, in $E$ spectrum one intense and three weak lines are ob-

\begin{tabular}{|c|c|c|c|}
\hline & $x x$ & yy & $z Z$ \\
\hline$A_{1}\left(A_{1}\right)$ & $\left(2 a+\Delta_{a}\right) \sqrt{2}$ & $\left(2 a+\Delta_{a}\right) \sqrt{2}$ & $2 a \sqrt{2}$ \\
\hline$A_{1}\left(F_{2}(x)\right)$ & $\delta_{c} \sqrt{2}$ & $\delta_{c} \sqrt{2}$ & $-2 \delta_{c} \sqrt{2}$ \\
\hline$A_{1}(E(1))$ & $-b \sqrt{2}$ & $-b \sqrt{2}$ & $2 b \sqrt{2}$ \\
\hline$A_{1}(E(2))$ & $b \sqrt{6}$ & $b \sqrt{6}$ & $-2 b \sqrt{6}$ \\
\hline$B_{1}\left(A_{1}\right)$ & $\Delta_{a} \sqrt{2}$ & $-\Delta_{a} \sqrt{2}$ & \\
\hline$B_{1}(E(1))$ & $-3 b \sqrt{2}$ & $3 b \sqrt{2}$ & \\
\hline$B_{1}(E(2))$ & $b \sqrt{6}$ & $-b \sqrt{6}$ & \\
\hline \multirow[t]{2}{*}{$B_{1}\left(F_{2}(x)\right)$} & $-\delta_{c} \sqrt{2}$ & $\delta_{c} \sqrt{2}$ & \\
\hline & $x y=y x$ & $x z=z x$ & $y z=z y$ \\
\hline$B_{2}\left(F_{2}(z)\right)$ & $2 d \sqrt{2}$ & & \\
\hline$B_{2}\left(F_{2}(y)\right)$ & $2 \delta_{d} \sqrt{2}$ & & \\
\hline$E(E(2))$ & & $2 \delta_{b}$ & $2 \delta_{b}$ \\
\hline$E\left(F_{2}(x, y)\right)$ & & $(d+c) \sqrt{2}$ & $(d+c) \sqrt{2}$ \\
\hline$E\left(F_{2}(x, y)\right)$ & & $(d-c) \sqrt{2}$ & $(d-c) \sqrt{2}$ \\
\hline$E\left(F_{2}(z)\right)$ & & $-2 \delta_{d}$ & $-2 \delta_{d}$ \\
\hline
\end{tabular}
served that differ from the prediction of one weak line. It is remarkable that in the frequency range of $v_{1}$ mode no Raman signal of $E$ polarization is detected, that is in agreement with the prediction. The analysis allows $4 B_{1,2}$ modes

Table 5. Raman tensor components calculated for $A_{1}, E$ and $F_{2}$ modes of tetrahedral $\left(\mathrm{VO}_{4}\right)^{3-}$ complexes in $\mathrm{SrNi}_{2} \mathrm{~V}_{2} \mathrm{O}_{8}$ crystal. In the first column the symmetry of a free tetrahedron mode is indicated in parentheses 
with the most intense component in the frequency region of the $v_{3}$ mode. Experimentally two intense and four weak lines are detected. One of the intense lines is close to the $v_{1}$ frequency. It may mean that $\Delta_{a}$ term of the molecular polarizability is essential enough. In diagonal $z z$ polarization ( $A_{1}$ modes) there are two intense enough lines close to frequencies of $v_{1}$ and $v_{3}$ modes of free $\mathrm{VO}_{4}$ tetrahedron. The analysis predicts less intense line due to $\delta_{C}$ term originated from the tetrahedra tilt. Its comparable with $A_{1}\left(v_{1}\right)$ mode intensity can not be explained in the proposed simple model where crystal polarizability is originated from the molecular polarizability only. Real situation may be too different from such a rough estimation. It means, for instance, that some of these internal modes of the molecular complexes may strongly polarize the crystal surrounding. Nevertheless the model provides evidence that a large number of $\left(\mathrm{VO}_{4}\right)^{3-}$ complexes situated in the crystal structure may lead to the interferential quenching of their polarizability, resulting in a smaller number of intense lines observed in the Raman spectra indeed.
As for $E\left(v_{2}\right)$ and $F_{2}\left(v_{4}\right)$ modes of the tetrahedral $\left(\mathrm{VO}_{4}\right)^{3-}$ complexes, the analysis predicts $3 A_{1}+5 B_{1,2}+4 E$ lines in the Raman spectrum of the crystal, including those with small intensity. Remembering the group theory prediction for these modes, $5 A_{1}+10 B_{1,2}+10 E$ (Table 1 ), we get about twice less detectable lines of this origin.

\subsection{Modes related to $\mathrm{Ni}^{2+}$ and $\mathrm{Sr}^{2+}$ degrees of freedom}

$\mathrm{Sr}^{2+}$ ions occupy positions with local symmetry $C_{2}$ in the crystal. Formally, $1 A_{1}+1 A_{2}+1 B_{1}+1 B_{2}+4 E$ modes are caused by their degrees of freedom (Table 1 ). The coordination of every $\mathrm{Sr}^{2+}$ ion consists of 8 oxygens. It turned out that the surrounding ligands form approximately higher symmetry, than $C_{2}$ Wyckoff position for $\mathrm{Sr}^{2+}$. The symmetry is close to $C_{2 v}$ with principal axis along $z$ direction. Figure 6 clearly shows permissibility of that approximation.

Polarizability tensors connected with the motions of $\mathrm{Sr}^{2+}$ ions in their positions are the following:

$$
\begin{aligned}
& \alpha_{z, 1,2}\left(A_{1}\right)=\left(\begin{array}{lll}
a & & \\
& b & \\
& & c
\end{array}\right), \\
& \alpha_{x, 1,2}\left(B_{1}\right)=\left(\begin{array}{ll} 
& e \\
e &
\end{array}\right) \text {, } \\
& \alpha_{y, 1,2}\left(B_{2}\right)=\left(\begin{array}{lll} 
& & \\
& f &
\end{array}\right), \\
& \alpha_{z, 3,4}\left(A_{1}\right)=\left(\begin{array}{lll}
b & & \\
& a & \\
& & c
\end{array}\right) \text {, } \\
& \alpha_{x, 3,4}\left(B_{1}\right)=\left(\begin{array}{ll} 
& f \\
&
\end{array}\right) \text {, } \\
& \alpha_{y, 3,4}\left(B_{2}\right)=\left(\begin{array}{ll} 
& e \\
e
\end{array}\right),
\end{aligned}
$$

(a)

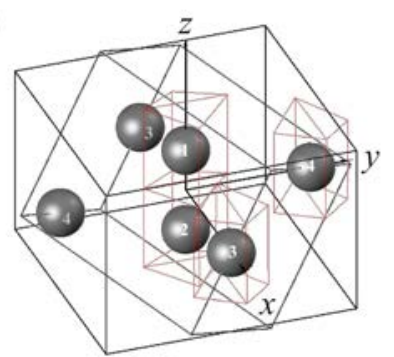

(b)

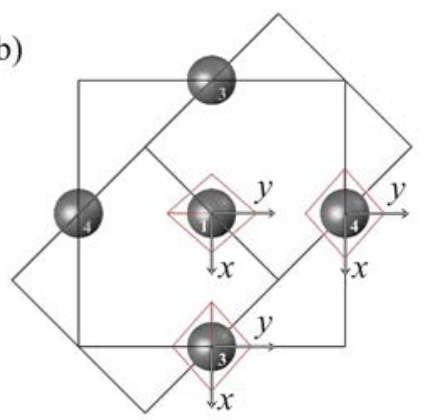

Fig. 6. (Color online) Structure of the $\mathrm{Sr}^{2+}$ coordination environment (a), and their projection on the basal plane (b) in $\mathrm{SrNi}_{2} \mathrm{~V}_{2} \mathrm{O}_{8}$ according to Refs. 20, 23. Atoms numeration and local coordinate systems are used in calculations.

The numeration follows Fig. 6. Symbols in parentheses relate to the $C_{2 v}$ point group irreps. Results of summations of expressions (7) through the normal modes originating from $\mathrm{Sr}^{2+}$ degrees of freedom in the crystal are presented in Table 6. As can be seen only $1 A_{1}+1 B_{1}+2 E \mathrm{Sr}^{2+}$ modes are expected to be intense in Raman. A deficit contains $1 B_{2}+2 E$ modes. Notably, tensors (7) do not contain $x y$ components at all, so there are no sources to produce Raman intensity of $B_{2}$ mode. This is a consequence of the actual lack of rotation around the $z$ axis of the oxygen polyhedron surrounding the $\mathrm{Sr}^{2+}$ ion.

Table 6. Raman tensor components calculated for $\mathrm{Sr}^{2+}$ and $\mathrm{Ni}^{2+}$ degrees of freedom in the $\mathrm{SrNi}_{2} \mathrm{~V}_{2} \mathrm{O}_{8}$ crystal. Labels $x, y, z$ in the first column represent directions of the ions shifts

\begin{tabular}{c|c|c|c}
\hline \hline & $x x$ & $y y$ & $z z$ \\
\hline \hline$A_{1}, z$ & $(a+b) \sqrt{2}$ & $(a+b) \sqrt{2}$ & $c 2 \sqrt{2}$ \\
$B_{1}, z$ & $(a-b) \sqrt{2}$ & $-(a-b) \sqrt{2}$ & \\
\hline \hline & $x y=y x$ & $x z=z x$ & $y z=z y$ \\
\hline \hline$E, x$ & & $(e+f) \sqrt{2}$ & \\
$E, y$ & & & $(e+f) \sqrt{2}$ \\
\hline$E, x$ & & $(e-f) \sqrt{2}$ & \\
$E, y$ & & & $(e-f) \sqrt{2}$ \\
\hline \hline
\end{tabular}




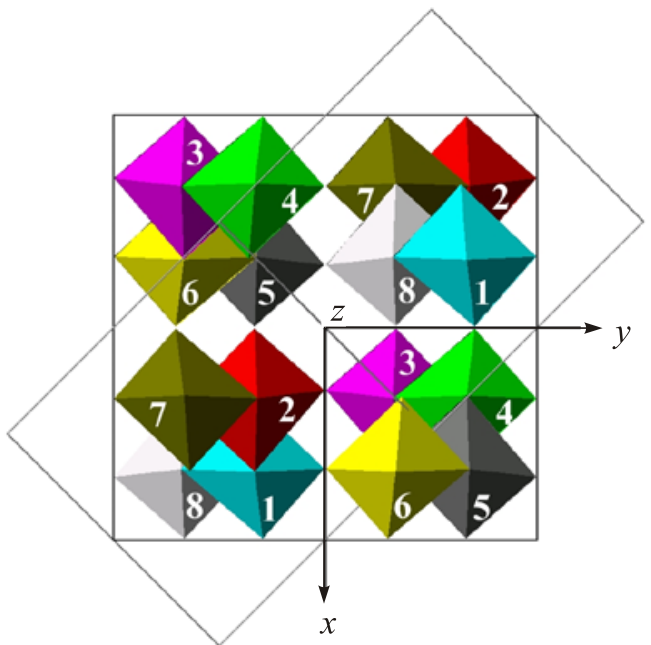

Fig. 7. (Color online) Structure of the $\mathrm{Ni}^{2+}$ coordination environment in $\mathrm{SrNi}_{2} \mathrm{~V}_{2} \mathrm{O}_{8}$ in the projection on basal plane according to Refs. 20, 23. Numeration of octahedrons is used in calculations.

Oxygen surroundings of $\mathrm{Ni}^{2+}$ in the structure of $\mathrm{SrNi}_{2} \mathrm{~V}_{2} \mathrm{O}_{8}$ looks like slightly distorted octahedron $[20,23]$ as shown in Fig. 7. Similarly to $\mathrm{Sr}^{2+}$ we will describe the symmetry of the $\mathrm{Ni}^{2+}$ coordination environment as $C_{2 v}$ in the first approximation. Eight complexes are divided into two groups 1, 4, 6, 7 and 2, 3, 5, 8 which possess polarizability tensors analogous to (7). And the results are obtained in the same manner as for $\mathrm{Sr}^{2+}$ degrees of freedom. In spite of twice greater number of $\mathrm{Ni}^{2+}$ ions in the crystal structure, symmetry limitations in the described model do reduce effectively this number, so the expected number of intense Raman lines proves to be the same as for the case of $\mathrm{Sr}^{2+}$ ions (Table 6). Effective symmetry rise in this case leads to a bigger deficit of intense Raman lines. It contains for $\mathrm{Ni}^{2+}$ degrees of freedom $2 A_{1}+2 B_{1}+3 B_{2}+4 E$.

Summing up the total proposed deficit of Raman lines in the "lattice" frequency region, we obtain $4 A_{1}+11 B_{1,2}+12 E$. This result is in a good agreement with experimental observations which estimate a lack of Raman lines in this region as $3 A_{1}+15 B_{1,2}+15 E$. Notice, that the proposed analysis does not include exploration of the rotational and translational degrees of freedom of $\mathrm{VO}_{4}$ tetrahedra which probably may produce extremely weak Raman signals too.

\section{Summary}

Vibrational Raman spectra of $\mathrm{SrNi}_{2} \mathrm{~V}_{2} \mathrm{O}_{8}$ are investigated in the $10-1000 \mathrm{~cm}^{-1}$ frequency range at various temperatures 7-300 K. There is no evidence of a structural phase transition in the investigated temperature range. Vibrational spectrum of the crystal naturally divides into two frequency ranges, namely "lattice" $\left(50-500 \mathrm{~cm}^{-1}\right)$ and internal $v_{1,3}$ modes of $\left(\mathrm{VO}_{4}\right)^{3-}$ tetrahedral complex $\left(700-900 \mathrm{~cm}^{-1}\right)$. In the low-frequency range $11 A_{1}+15 B_{1,2}+16 E$ modes are detected versus $14 A_{1}+30 B_{1,2}+31 E$ allowed by the symmetry. In the highfrequency range there are $4 A_{1}+6 B_{1,2}+4 E$ modes observed versus $4 A_{1}+8 B_{1,2}+8 E$ allowed. A big deficit of the observed Raman lines is explained by a simple model which takes into account the higher symmetry of the complexes in the crystal structure than it is given by the position symmetry. Such "symmetrization" leads to interferential quenching of certain modes, which takes place due to big number of the identical molecules and ions in the $\mathrm{SrNi}_{2} \mathrm{~V}_{2} \mathrm{O}_{8}$ structure.

1. I. Affleck, J. Phys.: Condens. Matter 1, 3047 (1989).

2. A. Smirnov and V. Glazkov, J. Exp. Theor. Phys. 105, 861 (2007).

3. F.D.M. Haldane, Phys. Rev. Lett. 50, 1153 (1983)

4. F.D.M. Haldane, Phys. Lett. A 93, 464 (1983).

5. M. Takahashi, Phys. Rev. Lett. 62, 2313 (1989).

6. O. Golinelli, T. Jolicoeur, and R. Lacaze, J. Phys.: Condens. Matter 5, 1399 (1993).

7. S.R. White and I. Affleck, Phys. Rev. B 77, 134437 (2008).

8. S. Ma, C. Broholm, D.H. Reich, B.J. Sternlieb, and R.W. Erwin, Phys. Rev. Lett. 69, 3571 (1992).

9. G. Xu, G. Aeppli, M.E. Bisher, C. Broholm, J.F. DiTusa, C.D. Frost, T. Ito, K. Oka, R.L. Paul, 10. H. Takagi, and M.M.J. Treacy, Science 289, 419 (2000).

10. P. Lemmens, G. Güntherodt, and C. Gros, Phys. Rep. 375, 1 (2003).

11. P.E. Sulewski and S.-W. Cheong, Phys. Rev. B 51, 3021 (1995).

12. T. Sakai and M. Takahashi, Phys. Rev. B 42, 4537 (1990).

13. A. Zheludev, T. Masuda, I. Tsukada, Y. Uchiyama, K. Uchinokura, P. Böni, and S.-H. Lee, Phys. Rev. B 62, 8921 (2000).

14. A. Zheludev, T.Masuda, K. Uchinokura, and S.E. Nagler, Phys. Rev. B 64, 134415 (2001).

15. Y. Uchiyama, Y. Sasago, I. Tsukada, K. Uchinokura, A. Zheludev, T. Hayashi, N. Miura, and P. Böni, Phys. Rev. Lett. 83, 632 (1999).

16. A.K. Bera and S.M. Yusuf, Phys. Rev. B 86, 024408 (2012).

17. B. Pahari, K. Ghoshray, R. Sarkar, B. Bandyopadhyay, and A. Ghoshray, Phys. Rev. B 73, 012407 (2006).

18. Z. He and Y. Ueda, J. Phys. Soc. Jpn. 77, 013703 (2008).

19. A.K. Bera, B. Lake, A.T.M.N. Islam, B. Klemke, E. Faulhaber, and J.M. Law, Phys. Rev. B 87, 224423 (2013).

20. A.K. Bera, B. Lake, A.T.M.N. Islam, O. Janson, H. Rosner, A. Schneidewind, J.T. Park, E. Wheeler, and S. Zander, Phys. Rev. B 91, 144414 (2015).

21. A.K. Bera, B. Lake, A.T.M.N. Islam, and A. Schneidewind, Phys. Rev. B 92, 060412R (2015).

22. Z. Wang, M. Schmidt, A.K. Bera, A.T.M.N. Islam, B. Lake, A. Loidl, and J. Deisenhofer, Phys. Rev. B 87, 104405 (2013).

23. R. Wichmann and H. Müller-Buschbaum, Rev. Chim. Miner. 23, 1 (1986).

24. N. Weinstock, H. Schulze, and A. Müller, J. Chem. Phys. 59, 5063 (1973).

25. E. Anachkova, M. Gospodinov, P. Svestarov, T. Milenov, A. Nikolov, V. Tasev, Y. Markov, M. Limonov, G. Bruchman, J. Mol. Struct. 219, 31 (1990). 
26. J.D. Pless, H.-S. Kim, J.P. Smit, X. Wang, P.C. Stair, and K.R. Poeppelmeier, Inorg. Chem. 45, 514 (2006).

27. R.L. Frost, S.J. Palmer, J. Čejka, J. Sejkora, J. Plášil, S. Bahfenne, and E.C. Keeffe, J. Raman Spectrosc. 42, 1701 (2011).

28. N. Zhang, J. Wang, X. Hu, H. Zhang, C.C. Santos, A.P. Ayala, and I. Guedes, J. Solid State Chem. 184, 905 (2011).

29. V. Panchal, S. López-Moreno, D. Santamaría-Pérez, D. Errandonea, F.J. Manjón, P. Rodríguez-Hernandez, A. Muñoz, S.N. Achary, and A.K. Tyagi, Phys. Rev. B 84, 024111 (2011).

30. L. Rghioui, L. Benarafa, J. El Jastimi, A. El Hajji, A. Lorriaux, and F. Wallart, J. Mater. Environ. Sci. 3 (1), 58 (2012).

31. W.P. Griffith and T.D. Wickins, J. Chem. Soc. (A): Inorg. Phys. Theor. 1087 (1966).
32. R.D. Shannon, Acta Cryst. A 32, 751 (1976).

33. A. de Andres, J.L. Martinez, R. Saez-Puche, and A. SalinasSanchez, Solid State Commun. 82, 931 (1992).

34. G. Gou, I. Grinberg, A.M. Rappe, and J.M. Rondinelli, Phys. Rev. B 84, 144101 (2011).

35. C.M. Julien, A. Mauger, K. Zaghib, R. Veillette, and H. Groult, Ionics 18, 625 (2012).

36. V. Gnezdilov, V. Kurnosov, P. Lemmens, Yu. Pashkevich, A.K. Bera, A.T.M.N. Islam, and B. Lake, Magnetic Excitations in the Symmetry Protected Topological Quasi-One-Dimensional Haldane Phase of $\mathrm{SrNi}_{2} \mathrm{~V}_{2} \mathrm{O}_{8}$, to be published. 